\title{
Cancer in Sri Lanka; trends, care and outcomes
}

\author{
Sanjeewa Seneviratne \\ Department of Surgery, Faculty of Medicine, University of Colombo, Sri Lanka
}

Keywords: Breast cancer; colorectal cancer; thyroid cancer; cancer registration; quality of care

\begin{abstract}
\section{Introduction}

The burden of cancer is increasing rapidly in Sri Lanka. However, limited data are available on incidence trends, diagnosis, treatment or outcomes of cancer in Sri Lanka. This oration encompasses a series of studies undertaken,

- To describe the trends in incidence

- To analyse stage at diagnosis, treatment and outcomes

- To describe the long-term quality of life [QOL] after completion of treatment of cancer in Sri Lanka
\end{abstract}

\section{Methods}

Three different strategies were undertaken.

- Incidence and trends - based on data published by the Sri Lanka National Cancer Registry [SLCR] during 2001-2010 for breast, thyroid and colorectal cancers. Joinpoint regression analysis was used to calculate changes over time.

- Diagnosis, treatment and outcomes - patients with breast and colorectal cancer registered between $01 / 01 / 2016$ and $31 / 12 / 2019$ for treatment at the Apeksha hospital, Maharagama were included.

- QOL - analysed using validated EORTC QLQ-C30 and QLQ-BR23 questionnaires in a cohort of women who have completed treatment for breast cancer.

\section{Results}

Significant increases in incidence were observed for all cancers which ranged between $2.5 \%$ to $8.5 \%$ per annum.

Apeksha hospital data included 4,185 breast and 1,985 colorectal cancers. While most of the cancer therapies appeared to have followed accepted guidelines, lower use of some of the adjuvant therapies is concerning.

Correspondence: Sanjeewa Seneviratne

E-mail: sanjeewa@srg.cmb.ac.lk

(iD) https://orcid.org/0000-0003-0310-1387

Received: 29-11-2020 Accepted: 25-12-2020

DOI: http://doi.org/10.4038/sljs.v38i3.8772
The QOL mean scores for sexual functioning, sexual enjoyment, systemic therapy, breast symptoms, arm symptoms, and hair loss assessed by the QLQ-BR23 were found to be poor with scores of 18.3, 33.3, 30.5, 16.2, 23.4 and 32.7 out of 100 , respectively.

\section{Conclusions}

Analysis of SLCR data confirmed the rise in the incidence of breast, colorectal and thyroid cancers. These findings may help predict future trends in incidence and implement strategies to control the incidence of these cancers. Disease and treatment patterns identified will be useful to plan future strategies to deal with these cancers more effectively.

\section{Introduction}

Cancer is the second most common cause of death in Sri Lanka [1]. Together with its rapidly aging population, cancer will have an increasingly major role in Sri Lanka's health system [2].

Researching into cancer incidence, trends, management, outcomes and disparities are important to identify areas where changes could be implemented to reduce the burden of cancer through measures to reduce incidence, early detection and better treatment strategies. However, a major limitation remains the lack of comprehensive information on incidence, trends, diagnostic and treatment pathways and its association with cancer outcomes which avert the identification of areas which are amenable to such interventions.

The National Cancer Control Program [NCCP] maintains the Sri Lanka Cancer Registry [SLCR]. NCCP data include all cancers treated at national cancer treatment centres and data from other major private and government hospitals, and pathology laboratories. This registry mainly contains sociodemographic and histologic data while staging data for some cancers are also included. In recent years, the SLCR has commenced collecting mortality statistics from death registries of hospitals in some provinces, but still remains largely incomplete [3]. Currently there is no systematic process to gather outcome data of any cancer in Sri Lanka. 
A series of studies were undertaken;

- To describe the incidence trends of selected common cancers in Sri Lanka,

- To analyse patterns of cancer stage at diagnosis, treatment and outcomes

- To describe the long-term quality of life [QOL] after completion of treatment for cancer in Sri Lanka

\section{Methods}

Three different strategies were undertaken.

- Incidence and trends - Based on data published by the NCCP for the period between 2001 and 2010.

- Diagnosis, treatment and outcomes - Patients with breast and colorectal cancer registered between 01/01/2016 and 31/2/2019 at the Apeksha Hospital, Maharagama

- QOL - was analysed using validated EORTC QLQC30 and QLQ-BR23 questionnaires in a cohort of women who have completed treatment for breast cancer [4].

\section{Incidence and trends}

\section{Study Population}

Details of all patients with breast, colorectal and thyroid cancers diagnosed between 01/01/2001 and 31/12/2010 were extracted from the publications of cancer incidence data of Sri Lanka by the NCCP [5].

\section{Analysis}

Age standardized rates of all cancers per 100,000 population were calculated using WHO age standardized populations including for age categories and by gender [6]. Joinpoint regression analysis was used to identify points where a statistically significant change over time in linear slope of the trend occurred [7].

\section{Diagnosis, treatment and cancer outcomes Study population}

A cohort of patients seeking care for breast and colorectal cancer was established at the Apeksha Hospital. Collected data included detailed patient demographics, information on cancer diagnosis, treatments [surgery, chemotherapy and radiation therapy] and outcomes [disease free survival, overall survival and recurrence].

\section{Developing the Electronic database, Data gathering and validation}

An online data repository was developed based on District Health Information System [DHIS2] software platform for data storage and analysis [http://cancersrilanka.space]. Data were gathered from January 1st, 2016 onwards, on new referrals to the Apeksha Hospital, Maharagama. All patients underwent a follow-up every six months.

\section{Analysis}

Categorical variables were described with numbers and percentages and continuous variables with mean/median with standard deviations. Survival analysis was done with KaplanMeier survival curves with disease recurrence as the censoring point. All analyses were performed using SPSS version 24

\section{Quality of life after treatment of cancer Study population}

All women with non-metastatic breast cancer who underwent surgery for breast cancer at the Professorial Surgical Unit, Colombo during 2015-2018 and completed a minimum of one year follow up were invited to participate in the study. Fiftyfour women who responded were analysed using the validated EORTC QLQ-C30 and QLQ-BR23 questionnaires [8].

\section{Analysis}

The data were coded and analysed according to the scoring protocol described in the EORTC QLQ-30 manual using SPSS version $24[8,9]$. Non-parametric tests including Chi square test and Mann-Whitney U test were used for univariate statistical analyses.

\section{Results}

\section{Incidence and trends}

\section{Breast cancer}

This study included 19,755 patients with newly diagnosed primary invasive breast cancer over the study period [10]. The overall mean age of the patients was 53.1 years [median 52 years].

The WHO age standardized incidence of breast cancer has increased from 9.2 in 2001 [95\% CI 8.17-9.62] to 12.9 per 100,000 in 2010 [95\% CI 12.4-13.5]; a 1.4-fold increase, [p $<0.05$ for trend] [Figure 1]. This is an EAPC in incidence of $4.4[95 \%$ CI 3.3-5.4] over this period.

Results of the Joinpoint analysis of incidence time trends by age, gender and histology type are shown in Table 1.

Only a modest increase in the incidence of breast cancer was observed among women younger than 50 years [from 32.0 to 39.6 per 100,000; $\mathrm{EAPC}=2.3,95 \%$ CI 1.1-3.5, $\mathrm{p}<0.05$ ], while the increase was substantially greater among women older than 50 years [ from 50.4 to 76.9 per 100,000 ; $\mathrm{EAPC}=5.5,95 \%$ CI 4.1-7.0, $\mathrm{p}<0.05]$ [Figure 2]. 


\section{Colorectal cancer}

A total of 7,694 colorectal cancers were diagnosed over the study period . There were 3,849 [50.5\%] males and 3,767 [49.4\%] females diagnosed of colorectal carcinoma with a male to female ratio of 1.02:1. The overall mean age of study patients was 58.7 years [median 59 years], the mean age in males was 59.1 [median 60 years] and in females was 58.0 [median 58 years]. The commonest site of colorectal cancer was seen in the rectum [51.1\%] followed by sigmoid colon [12.1\%] and recto sigmoid junction [4.8\%].

The WHO age standardized incidence of colorectal cancer in Sri Lanka has increased significantly from 2.9 per 100,000 in 2001 [95\% CI: $2.64-3.16$ ] to 6.08 per 100,000 in 2010 [95\% CI: 5.71-6.44] ; a 2.1-fold increase [ $<<0.05$ for trend]. This increase translates into an estimated annual percentage change [EAPC] of 8.9 [95\% CI 7.5-10.4] [Table 2]. The proportional increase in incidence was slightly greater for females [from 2.8 to 5.6; a 2.0-fold increase, EAPC: 9.4 [95\% CI: 7.7-11.2], [p $<0.05$ for trend] compared with males [from 3.02 to 6.62 ; a 2.2-fold increase, EAPC: 8.5 [95\% CI: 6.9 10.2 ], $\mathrm{p}<0.05$ for trend].

\section{Thyroid cancer}

This study included a total of 7,681 thyroid malignancies diagnosed over the 10-year study period [12]. The commonest histological type was papillary $[\mathrm{n}=5,302,69 \%]$ followed by follicular $[\mathrm{n}=1,411,18.4 \%]$, medullary $[\mathrm{n}=138,1.8 \%]$ and anaplastic $[n=285,3.71 \%]$. The majority of the cancers were in females $[n=6,166,80.3 \%]$ with a male to female ratio of 1 : 4.07. Mean age of study patients was 43.8 years [median 44 years].

Results of thyroid cancer incidence in Sri Lanka with Joinpoint analysis of trends by gender, age group and histology subtype are shown in Table 3.

The WHO age standardized incidence of thyroid cancer in Sri Lanka was observed to have increased significantly from 2.44 per 100,000 in 2001 [ $95 \%$ CI: $2.21-2.67$ ] to 5.16 per 100,000 in 2010 [95\% CI: 4.85-5.47]; a 2.1-fold increase [ $\mathrm{p}<0.05$ for trend] [Figure 3]. This is an EAPC of 8.2 [95\% CI 5.9-10.5]. The proportional increase in incidence was greater for females [from 3.61 to 8.06; a 2.23-fold increase, $\mathrm{p}<0.05$ for trend] compared with males [from 1.24 to 2.15 ; a 1.73 -fold increase, $p<0.05$ for trend] [Figure 3].A greater part of the increase in thyroid cancer incidence is attributable to an increase in incidence of papillary thyroid cancer, which has increased from 1.64 to 3.61 per 100,000; a 2.2-fold increase $[\mathrm{p}<0.05$ for trend].

\section{Diagnosis, treatment and outcomes}

This study included all breast $[\mathrm{n}=4,185]$ and colorectal $[\mathrm{n}=1,195]$ cancer patients who registered for adjuvant treatment at the National Cancer Institute, Maharagama during the period between 01st January 2016 and 31st December 2019.

\section{Breast cancer}

Distribution of patient and tumour characteristics of the study population is shown in Table 4. The mean age of the women was 55.7 years [standard deviation [SD] 11.6, median 56 years, range $20-93$ years].

Just over two thirds [67.8\%] of the cancers were of early stage [stage I \& II] at diagnosis [Table 4]. Of the patients with data available on all biological and receptor characteristics $[\mathrm{n}=3291,78.6 \%]$ tumour subtype analysis was done. Of those $58 \%$ were luminal A type cancers while the rate of triple negative cancers was $19.6 \%$.

Treatment characteristics of the women included in the analysis is shown in Table 5 .

Of the total population of women, $14.2 \%$ had received neoadjuvant chemotherapy [NACT]. Adjuvant therapy characteristics were analysed in women who have completed adjuvant chemotherapy and radiotherapy $[n=3,573]$. Approximately two thirds [67.7\%] have received adjuvant chemotherapy. Radiotherapy to the breast, chest wall or nodal basins was received by nearly $60 \%[n=2,131]$. Receipt of radiotherapy by different types of surgery to the breast was analysed for women who were diagnosed during 2016-2018 as some patients diagnosed in 2019 are still undergoing chemotherapy and were yet to receive radiotherapy. Of the 3,197 eligible patients, 2,281 [71.3\%] and 733 [22.9\%] had undergone mastectomy and wide local excision, respectively. Of the mastectomy patients, 1,425 [62.5\%] had received radiotherapy while $540[73.7 \%]$ from the wide local excision group had received radiotherapy [data not shown].

We analyzed the concordance between guideline recommendations for use of adjuvant therapy for nonmetastatic breast cancer and actual rates of delivery [Table 6] $[13,14]$. Rate of chemotherapy delivery [neoadjuvant or adjuvant] was $89.3 \%$ for women with node positive breast cancer. Approximately $75 \%$ eligible women [i.e., following breast conserving therapy or with $3+$ positive lymph nodes] have received adjuvant radiotherapy. Endocrine therapy has been initiated in $85 \%$ of women with hormone receptor positive breast cancer while rate of trastuzumab use was $49 \%$ among women with HER2 positive breast cancer. 
Recurrence free survival rates were analyzed for nonmetastatic breast cancers which were $96 \%, 90 \%$ and $74 \%$ for stage I, II and III cancers, respectively at 3-years.

\section{Colorectal cancer}

Of the 1,195 patients included the mean age was 53.3 years [range 18 to 90 years, median 62.3, SD 11.8]. There was a slightly higher number of males $[\mathrm{n}=605,51 \%]$ than females $[\mathrm{n}=590,49 \%$ ]. Colorectal cancer stage at diagnosis is shown in Table 7.

Overall, the use of adjuvant chemotherapy has been used in $87.5 \%$ patients out of which $28 \%$ have received it prior to surgery [neoadjuvant]. Almost all patients [99\%] for whom chemotherapy was absolutely indicated according to guidelines have received chemotherapy.

\section{Quality of life}

Fifty-four female patients were included in the study with a mean age of 59.0 years [median 60 years, range 36-81] with $61.1 \%$ [n=33] being less than 60 years. Majority of the women were postmenopausal $[85.2 \% \%, \mathrm{n}=46]$. A majority $[61.1 \%, \mathrm{n}=33]$ underwent mastectomy as the primary surgery and the rest [45\%, $\mathrm{n}=19]$ underwent BCS. Axillary node dissection was performed in $59.2 \%[\mathrm{n}=32]$ and the rest only a sentinel lymph node biopsy.

The mean EORTC QLQ-C30 and QLQ-BR23 scores are shown in Table 9.

\section{Discussion}

\section{National Cancer Registry Data}

The studies based on the Sri Lanka National Cancer Registry [SLCR] have shown a steady increase in the incidence of nearly all of the included common cancers over the 10-year study period. Overall, the rates of increase in incidence ranged between $2.5 \%$ to $8.5 \%$ per year.

There are many possible reasons for the observed increases in cancer incidence. One is the gradual increase in screen detected cancer [15]. Although Sri Lanka does not have national cancer screening programmes except for cervical cancer, many government and private institutions have started providing opportunistic cancer screening especially over the last decade [16]. Another likely contributor is better reporting and greater coverage of cancer data by the cancer registry [5].

Despite all these possible reasons for an 'artificial' increase in the incidence, it is likely that there has been a genuine increase in the incidence similar to many other developing countries [1]. Several factors have been proposed to be possible contributors towards this increase. These include westernization of lifestyle including increased consumption of processed and fatty food, sedentary lifestyle leading to increased obesity and increasing rates of alcohol and smoking [17].

In general, the highest incidence of cancers was seen in 60-70 age group. Further, the rate of rise in incidence was also observed to be higher in this age group. Sri Lanka has one of the fastest ageing populations in the world [18]. Older patients are more likely to have more comorbidities and a poorer survival from cancer [19]. Healthcare policy makers in the country need to consider all these factors in planning strategies, if they are to effectively deal with the increasing burden of cancer.

We propose several changes to improve the utility of data collected by the SLCR. First the coverage of cancer data collection needs to be improved. Introducing legislation, for example to make informing a cancer diagnosis compulsory to all pathologists or pathology laboratories are simple yet very effective ways to increase the completion. Further this could be used to improve the coverage additional data including the cancer stage. Thirdly, combining cancer data with cause of death data would provide accurate figures of cancer specific survival. Although this is more difficult as it requires a change in the system of death registration, such a change would be useful to identify the mortality not only of cancer, but of many other important diseases as well.

\section{Cancer data from the National Cancer Institute, Maharagama}

Although still in very early stages, this data collection project has shown useful results and has also shown the feasibility of collecting cancer data efficiently and cost effectively.

\section{Breast cancer}

To our knowledge, this is the largest published cohort of breast cancer patients in Sri Lanka and the most comprehensive especially in relation to treatment characteristics.

The proportion of early stage breast cancer in our study is comparable to the two previous studies Balawardana et al and Mudduwa et al who have observed $68 \%$ and $67 \%$ early breast cancers, respectively. This probably is a reflection of failure of the health system of the country to implement effective strategies aimed at early diagnosis of breast cancer.

Present study has shown a substantial increase in the rate of breast conservation surgery [22.7\%] as compared with the study by Balawardena et al where the proportion of breast conservative surgery was only $3 \%$ despite including a similar proportions of stage I and II cancers compared with the 
present study [20]. This probably reflects better access to mammography, surgeon expertise as well as greater awareness among women with breast cancer.

We identified considerable lapses in the concordance between guideline recommendations and the delivery of cancer care $[13,14]$. For instance, only $75 \%$ women with absolute indications for radiotherapy have received adjuvant radiotherapy. Difficulties and limitations in access, patient co-morbidities, socioeconomic factors and poor health literacy are some of the known factors that may have limited the use of optimum adjuvant therapy.

Government of Sri Lanka has initiated several strategies with the aim of improving access to treatments and the quality of care for patients with cancer in the country. For instance, a program has been implemented to procure linear accelerators and to station radiation oncology centres in each of the nine provinces of the country to improve the access to radiotherapy [20]. Further action is needed to improve the availability resources including chemotherapy, endocrine and targeted therapy to ensure easier access with minimal delays. In addition, multidisciplinary team [MDT] to discuss and provision of care may improve quality of care and ensure all patients receive standard guideline concordant care. Advances in information technology will allow smaller base hospitals to obtain specialist cancer advise by joining MDT meetings held at tertiary hospitals online.

There are several limitations in our study. Incomplete and missing data were identified mainly in relation to adjuvant therapy and treatment modalities. Nevertheless, this is thus far the largest and most comprehensive cohort of patients with breast cancer reported from Sri Lanka.

\section{Colorectal cancer}

In this study, we have described disease characteristics and treatment patterns in a cohort of patients with newly diagnosed colorectal cancer in Sri Lanka.

Advanced stage at diagnosis was one of the most prominent features with nearly $57 \%$ had locally advanced or metastatic disease at presentation. Use of adjuvant therapy appeared to have been well in concordance with the standard guidelines. For instance, over $99 \%$ of stage III colorectal cancers have received either neoadjuvant or adjuvant chemotherapy. However, the overall chemotherapy rate of $87.5 \%$ may point towards overuse of chemotherapy especially among patients with low risk stage I and II disease.
There are several limitations in this study too similar to breast cancer study. Incomplete and missing data were identified mainly in relation to adjuvant therapy and treatment modalities is a major limitation.

\section{Future}

Future plans for the data repository include coverage of cancers beyond breast and colorectal and expansion to include data from other national cancer treatment centres. Many of the Sri Lankan public sector hospitals are in the process of converting from paper based to electronic data recording systems. Hence, linkage of these databases in the future is realistic which would potentially allow access to many patient and cancer related information as well as follow-up data from these hospital databases.

Many potential challenges are anticipated which include trained manpower for data collection, and long-term project funding. While the challenges remain real, with wider participation of stakeholders including the relevant government organizations we believe it will be possible to overcome these challenges successfully.

\section{Quality of life}

This prospective cohort study evaluated the post-treatment long-term QOL in Sri Lankan female patients diagnosed with breast cancer has shown substantially poor QOL in sexual functioning and enjoyment, breast and arm symptoms and hair loss domains while the impact on global health status including physical, social and emotional functions were minimal.

According to our findings, the low sores in breast related symptoms measured by BR-23 seems to be a major contributing factor for the lower QOL in breast cancer patients. Taking this into consideration, it is necessary to take measures to address the burden of breast related symptoms of these patients following surgery as these are easily preventable with adequate care. The HRQL of these patients may be improved by simple measures such as addressing sexual issues by referring them for counselling and prescribing topical applications, offering physiotherapy to alleviate arm symptoms, provision of wigs to combat hair loss following treatment. Provision of regular contact with the patients through trained cancer care nurses to recognize these issues and provide advice may help improve QOL in these domains which ultimately will help improve overall QOL. 


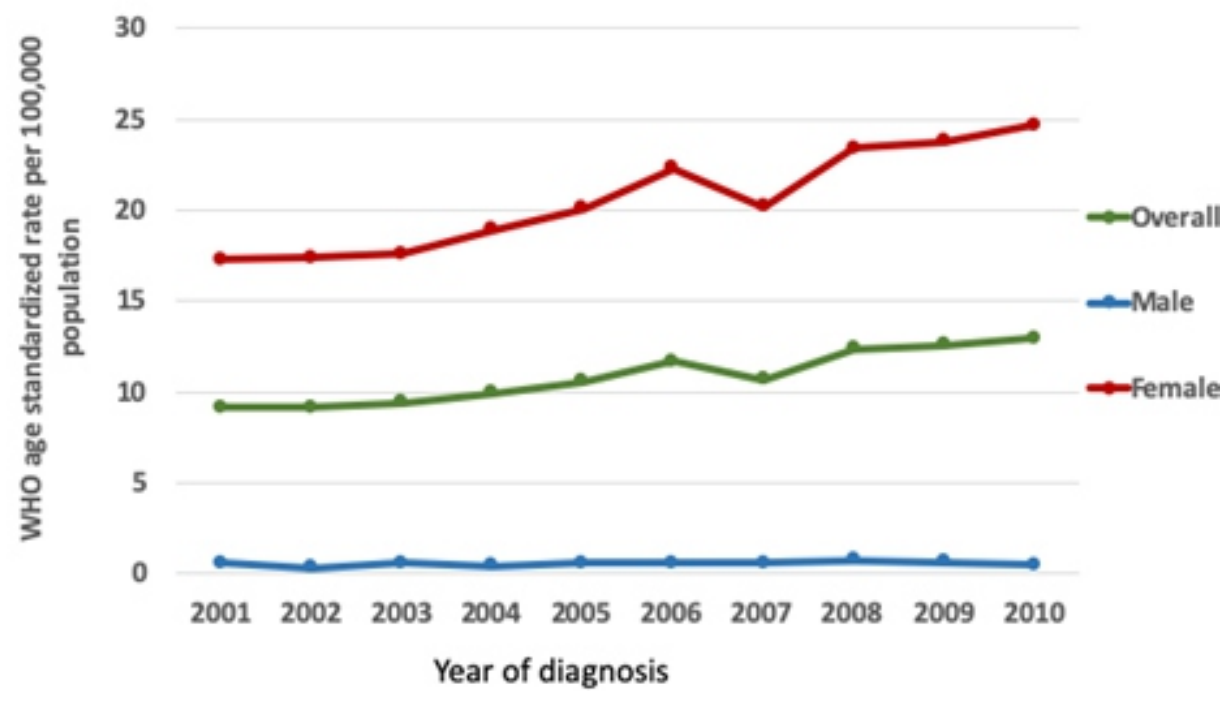

Figure 1. Trends in the incidence of breast cancer in Sri Lanka 2001-2010
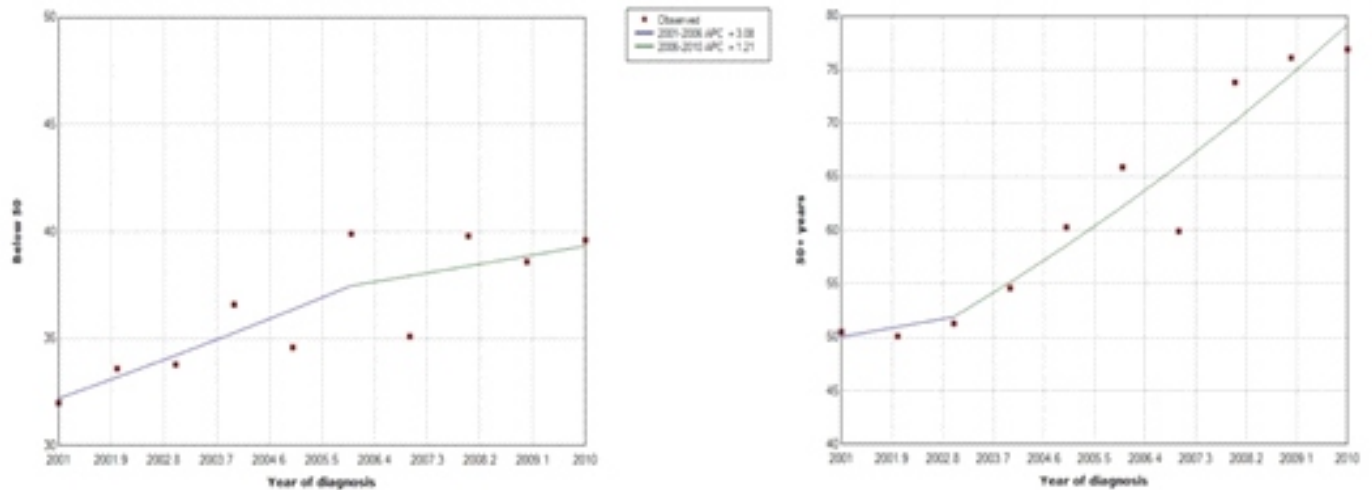

Figure 2. Joinpoint analysis of incidence of breast cancer by age category, $<50$ years versus $50+$ years
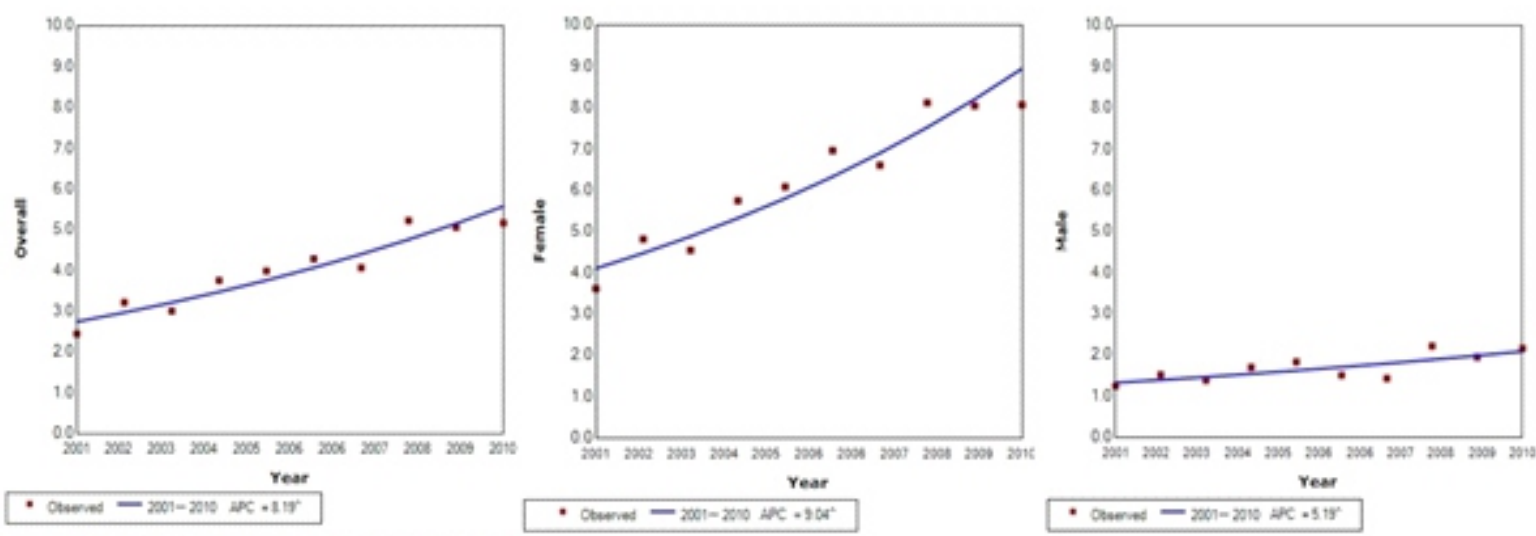

Figure 3. Joinpoint analysis of incidence of thyroid cancer by gender in Sri Lanka 2001-2010 


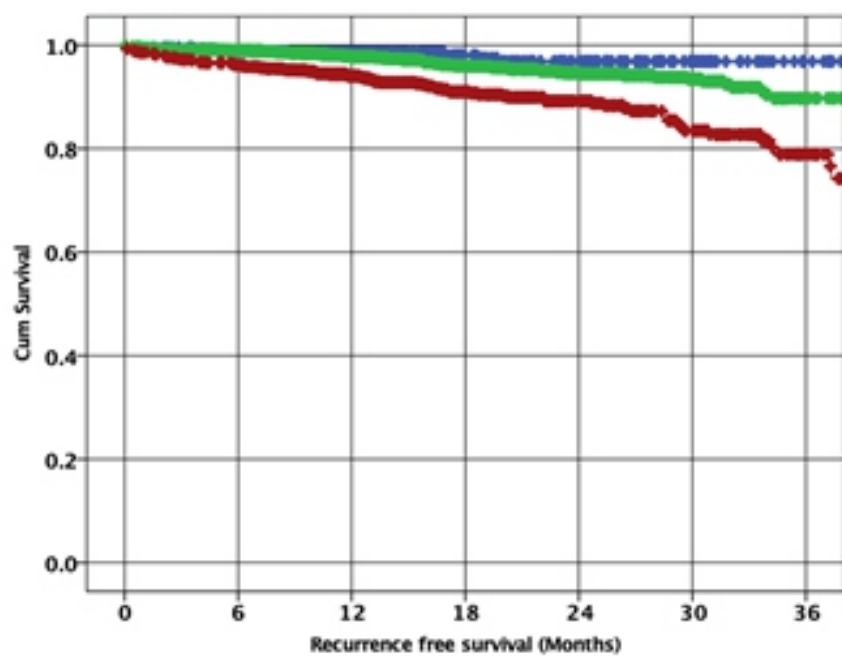

Stage I-96\%

Stage II $-\mathbf{9 0 \%}$

Stage III - 74\%

Figure 4. Recurrence free survival of non-metastatic breast cancer received treatment at Apeksha Hospital, 2016-2019

Table 1. Breast cancer incidence in Sri Lanka by gender and age group with Joinpoint analysis of Estimated Annual Percentage Change [EAPC] from 2001-2010

\begin{tabular}{|c|c|c|c|c|c|}
\hline & \multicolumn{2}{|l|}{2001} & \multicolumn{2}{|l|}{2010} & \multirow{2}{*}{$\begin{array}{l}\text { EAPC 2001-2010 } \\
\text { [95\% Cl] }\end{array}$} \\
\hline & $\mathbf{n}$ & Rate [95\% Cl] & $\mathbf{n}$ & Rate [95\% Cl] & \\
\hline \multicolumn{6}{|l|}{ Age group [years] } \\
\hline \multicolumn{6}{|l|}{ Female } \\
\hline$<20$ & 2 & 0.06 & 2 & 0.06 & 0 \\
\hline $20-39$ & 197 & 6.42 & 263 & 7.78 & $2.12^{*}$ \\
\hline $40-59$ & 952 & 44.6 & 1374 & 58.4 & $3.09^{*}$ \\
\hline $60+$ & 396 & 48.1 & 755 & 74.8 & $5.55^{*}$ \\
\hline Age standardized & 1547 & $17.3[16.5-18.2]$ & 2401 & $24.7\lceil 23.7-25.7\rceil$ & $4.4[3.3-5.5]^{*}$ \\
\hline $\begin{array}{l}\text { Overall age } \\
\text { standardised rate }\end{array}$ & 1591 & $9.17[8.17-9.62]$ & 2440 & $13.0[12.5-13.5]$ & $4.4[3.3-5.4]$ \\
\hline \multicolumn{6}{|l|}{ Histology type } \\
\hline \multicolumn{6}{|l|}{ Fernale } \\
\hline Ductal CA & 1221 & $13.6[12.9-14.4]$ & 2085 & $21.4\lceil 20.5-22.4\rceil$ & $5.2\lceil 2.8-7.8]^{*}$ \\
\hline Lobular CA & 74 & $0.8[0.6-1.0]$ & 55 & $0.6[0.4-0.7]$ & $-3.3[-10.4-19.2]$ \\
\hline Others & 94 & $1.0[0.8-1.3]$ & 119 & $1.2[1.0-1.5]$ & $2.4[-2.4-7.5]$ \\
\hline
\end{tabular}

*The EAPC is significant $[p<0.05]$ 
Table 2. Colorectal cancer incidence in Sri Lanka by gender and age group with joinpoint analysis 2001-2010

\begin{tabular}{llllll}
\hline & $\mathbf{n}$ & Rate [95\% Cl] & $\mathbf{n}$ & Rate [95\% Cl] & [95\% Cl] \\
\hline $\begin{array}{lllll}\text { Age group [years] } \\
\text { Male }\end{array}$ & & & & \\
$15-29$ & 10 & 0.35 & 12 & 0.43 & - \\
$30-44$ & 31 & 1.78 & 57 & 2.54 & - \\
$45-59$ & 92 & 8.76 & 179 & 11.48 & - \\
$60-74$ & 87 & 16.29 & 246 & 35.34 & - \\
$75+$ & 21 & 15.11 & 67 & 32.68 & \\
& & & & & \\
Age standardized & 241 & $3.02[2.64-3.4]$ & 562 & $6.62[6.07-7.16]$ & \\
Age group [years] & & & & & - \\
Female & & & & & - \\
15-29 & 4 & 0.14 & 6 & 0.22 & - \\
30-44 & 46 & 2.73 & 53 & 2.30 & - \\
$45-59$ & 95 & 9.79 & 177 & 10.83 & - \\
$60-74$ & 81 & 17.53 & 203 & 26.36 & $9.4[7.7-11.2]^{*}$ \\
$75+$ & 20 & 15.50 & 75 & 31.38 & \\
Age standardized & 246 & $2.8[2.5-3.2]$ & 514 & $5.6[5.1-6.1]$ & \\
Overall age & 487 & $2.9[2.64-3.16]$ & 1076 & $6.08[5.71-6.44]$ & \\
standardized rate & & & & & \\
\hline
\end{tabular}

*The EAPC is significant $[p<0.05]$

Table 3. Thyroid cancer incidence in Sri Lanka by gender, age group and histology subtype with Joinpoint analysis 2001-2010

\begin{tabular}{|c|c|c|c|c|c|}
\hline \multicolumn{6}{|l|}{ Age group [years] } \\
\hline \multicolumn{6}{|l|}{ Male } \\
\hline $20-39$ & 39 & 1.29 & 61 & 1.84 & $4.2[0.0-8.6]$ \\
\hline $40-59$ & 40 & 1.93 & 83 & 3.66 & $5.9[0.2-11.9]$ \\
\hline $60+$ & 26 & 3.16 & 52 & 5.77 & $5.3[0.6-10.2]$ \\
\hline Age standardized & 106 & $1.24[1.00-1.47]$ & 203 & $2.15[1.85-2.44]$ & $5.3[1.9-8.8]$ \\
\hline \multicolumn{6}{|l|}{ Age group [years] } \\
\hline \multicolumn{6}{|l|}{ Female } \\
\hline $\begin{array}{l}20-39 \\
40-59\end{array}$ & $\begin{array}{l}143 \\
127\end{array}$ & $\begin{array}{l}4.66 \\
5.95\end{array}$ & $\begin{array}{l}363 \\
348\end{array}$ & $\begin{array}{l}10.7 \\
14.8\end{array}$ & $\begin{array}{l}9.8[7.2-12.6] \\
9.2[6.3-12.2]\end{array}$ \\
\hline $60+$ & 54 & 5.90 & 99 & 9.81 & $4.1[0.8-8.5]$ \\
\hline Age standardized & 337 & $3.61[3.23-4.00]$ & 831 & $8.06[7.52-8.65]$ & $9.0[6.6-11.5]$ \\
\hline $\begin{array}{l}\text { Overall age } \\
\text { standardized rate } \\
\text { Histology type }\end{array}$ & 443 & $2.44[2.21-2.67]$ & 1034 & $5.16[4.85-5.47]$ & $8.2[5.9-10.5]$ \\
\hline \multicolumn{6}{|l|}{ [Overall] } \\
\hline Papillary & 304 & 1.64 [1.46-1.83] & 735 & $3.61[3.35-3.87]$ & $8.8[6.6-11.1]$ \\
\hline Follicular & 100 & $0.56[0.45-0.67]$ & 188 & $0.95[0.82-1.09]$ & $6.5[5.2-7.9]$ \\
\hline Other & 39 & $0.24[0.16-0.31]$ & 111 & $0.61[0.49-0.71]$ & $7.6[1.0-14.6]$ \\
\hline
\end{tabular}

*EAPC - Estimated Annual Percentage Change 
Table 4. Demographic and tumour characteristics of women with breast cancer diagnosed during 2016-2019 at the National Cancer Institute, Sri Lanka

\begin{tabular}{l}
\hline Characteristic \\
\hline Age category \\
440 \\
$40-49$ \\
$50-59$ \\
$60-69$ \\
$70+$ \\
Year of diagno \\
2016 \\
2017 \\
2018 \\
2019 \\
Stage category \\
I \\
II \\
III \\
IV
\end{tabular}

\section{Histology type}

$\begin{array}{lc}\text { Ductal CA } & 3479[88.4] \\ \text { Lobular CA } & 189[4.8] \\ \text { Mucinous CA } & 85[2.2] \\ \text { Papillary CA } & 51[1.3] \\ \text { Metaplastic CA } & 25[0.6] \\ \text { Other } & 108[2.7] \\ \text { Missing } & {[248]}\end{array}$

\section{Grade}

$\begin{array}{lc}\text { I } & 580[15.5] \\ \text { II } & 1880[50.3] \\ \text { III } & 1278[34.2] \\ \text { Missing } & {[447]}\end{array}$

\section{Subtype}

Luminal A

Luminal B

HER-2 enriched

Triple negative

Missing

575 [13.7]

2266 [54.1]

1249 [29.8]

95 [2.3]

968 [23.1]

1103 [26.4]

1133 [27.1]

981 [23.4]

[248]

Total
1910 [58.0]

428 [13.0]

308 [9.4]

$645[19.6]$

[894]
Table 5. Treatment characteristics of women with breast cancer diagnosed during 2016-2019 at the National Cancer Institute, Sri Lanka

\begin{tabular}{|c|c|}
\hline Type of treatment & $\mathbf{n}[\%]$ \\
\hline \multicolumn{2}{|l|}{ Surgery-Breast } \\
\hline Mastectomy only & $2825[67.6]$ \\
\hline Mastectomy + reconstruction & $36[0.9]$ \\
\hline Wide local excision & $948[22.7]$ \\
\hline No surgery & $365[8.7]$ \\
\hline Missing & {$[11]$} \\
\hline \multicolumn{2}{|l|}{ Surgery-Axilla } \\
\hline Sentinel LN biopsy & $833[20.0]$ \\
\hline Axillary clearance & $2914[69.8]$ \\
\hline No surgery & $426[10.2]$ \\
\hline Missing & {$[12]$} \\
\hline \multicolumn{2}{|l|}{ Neoadjuvant chemotherapy } \\
\hline Yes & $509[12.2]$ \\
\hline No & $3676[87.8]$ \\
\hline \multicolumn{2}{|l|}{ Adjurant chemotherapy } \\
\hline Yes & $2422[67.7]$ \\
\hline No & $1151[32.2]$ \\
\hline Not-started / not-completed & {$[612]$} \\
\hline \multicolumn{2}{|l|}{ Adjuvant radiotherapy } \\
\hline Yes & $2131[59.6]$ \\
\hline No & $1442[40.3\rceil$ \\
\hline Not-started / not-completed & {$[612]$} \\
\hline \multicolumn{2}{|l|}{ Adjuvant endocrine therapy } \\
\hline Yes & $2136[59.8]$ \\
\hline No & $1437[40.2]$ \\
\hline Not started & {$[612]$} \\
\hline
\end{tabular}


Table 6. Concordance between adjuvant treatments delivered to women with non-metastatic breast cancer diagnosed during 2016-2019 at the National Cancer Institute, Sri Lanka versus guideline* recommendations

*National Institute of Health and Care Excellence [NICE] and National Comprehensive Cancer Network [NCCN]

\begin{tabular}{|c|c|c|}
\hline Indicator & Groups & n [\%] \\
\hline Use of chemotherapy [CT] & CT indicated & $2119[100]$ \\
\hline $\begin{array}{l}\text { - CT should be delivered to patients with node-positive } \\
\text { disease }\end{array}$ & CT delivered & $1892[89.3]$ \\
\hline Use of adjuvant radiotherapy [RT] & RT indicated & $1280[100]$ \\
\hline $\begin{array}{l}\text { - RT should be delivered to patients after breast } \\
\text { conserving surgery and those with } 3+\text { positive LNs }\end{array}$ & RT delivered & $961[75.1]$ \\
\hline Use of adjuvant endocrine therapy [ET] & ET indicated & $2116[100]$ \\
\hline $\begin{array}{l}\text { - Adjuvant ET shouid be given to all patients with } E R+/-P R \\
\text { positive breast cancer }\end{array}$ & ET started & $1803[85.2]$ \\
\hline Use of adjuvant trastuzumab & Trastuzumab indicated & $720[100]$ \\
\hline $\begin{array}{l}\text { - Adjuvant trastuzumab should be given to all patients } \\
\text { with HER2 positive breast cancer }\end{array}$ & Trastuzumab delivered & $353[49.0]$ \\
\hline
\end{tabular}

Table 7. Stage distribution of breast cancer patients treated at National Cancer Institute, Maharagama 2016-2019

\begin{tabular}{lll}
\hline Indicator & Groups & $\mathbf{n ~ [ \% ] ~}$ \\
& & \\
\hline Use of chemotherapy [CT] & CT indicated & 588 \\
& & {$[100]$} \\
- CT shoulo be delivered to & CT delivered & 583 \\
patients with stage III & & {$[99.1]$} \\
disease & & \\
\hline
\end{tabular}

Table 8. Concordance between adjuvant treatments delivered to patients with colo-rectal cancer diagnosed during 20162019 at the National Cancer Institute, Sri Lanka versus guideline* recommendations

\begin{tabular}{llc}
\hline Stage & & $\mathbf{n}[\%]$ \\
\hline Stage category & 0 & $2[0.2]$ \\
& I & $165[13.8]$ \\
& II & $338[28.3]$ \\
& II & $588[49.2]$ \\
& Unknown & $100[8.4]$ \\
Year & 2016 & {$[2]$} \\
& 2017 & $245[20.5]$ \\
& 2018 & $228[19.1]$ \\
Histology & 2019 & $361[30.2]$ \\
& Adenocarcinoma & $361[30.2]$ \\
& Mucinous carcinoma & $995[94.5]$ \\
& Signet ring cell carcinoma & $46[4.3]$ \\
& Other & $7[0.6]$ \\
& Unknown & $5[0.5]$ \\
Differentiation & {$[142]$} \\
& Well & \\
& Moderate & $55[5.4]$ \\
& Poor & $916[91.3]$ \\
& Unknown & $32[3.1]$ \\
& & {$[192]$} \\
& & $1195[100]$ \\
\hline
\end{tabular}

*National Institute of Health and Care Excellence [NICE] and National Comprehensive Cancer Network [NCCN] 
Table 9. EORTC QLQ-C30 and QLQ-BR23 mean scores of women after a minimum of 1-year after completion of treatment for breast cancer in Sri Lanka [N=54]

\begin{tabular}{llccc}
\hline & Scales/ltems & Mean & Range & SD \\
\hline $\begin{array}{l}\text { Global health } \\
\text { status/ HRQL }\end{array}$ & 68.8 & $8.3-100$ & 27.1 \\
\hline QLQ-C30 & Physical function & 71.4 & $33.3-100$ & 19.78 \\
& Role function & 81.5 & $0-100$ & 20.13 \\
& Emotional function & 77.0 & $0-100$ & 26.59 \\
& Cognitive function & 80.2 & $33.3-100$ & 18.33 \\
& Social Function & 86.4 & $0-100$ & 24.24 \\
\hline QLQ-BR23 & Body image & 76.4 & $16.7-100$ & 25.99 \\
& Sexual functioning & 18.3 & $0-100$ & 23.509 \\
& Sexual enjoyment & 33.3 & $0-100$ & 32.33 \\
& Future perspective & 73.6 & $0-100$ & 34.81 \\
& Systemic therapy side & 30.5 & $0-90.5$ & 22.765 \\
& effects & & & \\
& Breast symptoms & 16.2 & $0-66.7$ & 14.058 \\
& Arm symptoms & 23.4 & $0-77.7$ & 20.32 \\
& Hair loss & 32.7 & $0-100$ & 39.87 \\
\hline
\end{tabular}

All authors disclose no conflict of interest. The study was conducted in accordance with the ethical standards of the relevant institutional or national ethics committee and the Helsinki Declaration of 1975, as revised in 2000 .

\section{References}

1. Ferlay J, Soerjomataram I, Ervik M, Dikshit R, Eser S, Mathers C, et al. GLOBOCAN 2012 v1.0, Cancer Incidence and Mortality Worldwide Lyon, France: International Agency for Research on Cancer; 2013 [Available from: http://globocan.iarc.fr.

2. Abeykoon A. Ageing and the health sector in Sri Lanka. Meeting the challenges calls for fresh thinking and focused strategies. Ceylon Medical Journal. 2000;45[2]:52-4. https://doi.org/10.4038/cmj.v45i2.7999

3. Gunasekera S, Seneviratne S, Wijeratne T, Booth CM. Delivery of cancer care in Sri Lanka. Journal of Cancer Policy. 2018;18:20-4. https://doi.org/10.1016/j.jcpo.2018.10.001

4. Jayasekara H, Rajapaksa LC, Brandberg Y. Measuring breast cancer-specific health-related quality of life in South Asia: psychometric properties of the Sinhala version of the EORTC QLQ-BR23. Quality of Life Research. 2008;17[6]:927-32. https://doi.org/10.1007/s11136-008-9359-9
5. National Cancer Control Programme Sri Lanka. Cancer Incidence Data, Sri Lanka 2001-2010. Ministry of Health; 2016.

6. Ahmad OB, Boschi-Pinto C, Lopez AD, Murray CJ, Lozano R, Inoue M. Age standardization of rates: a new WHO standard. Geneva: World Health Organization. 2001;9.

7. Joinpoint Regression Program V. Statistical Methodology and Applications Branch. Surveillance Research Program, National Cancer Institute, USA.May 2017.

8. Aaronson NK, Ahmedzai S, Bergman B, Bullinger M, Cull A, Duez NJ, et al. The European Organization for Research and Treatment of Cancer QLQ-C30: a quality-of-life instrument for use in international clinical trials in oncology. JNCI: Journal of the National Cancer Institute. 1993;85[5]:365-76.

https://doi.org/10.1093/jnci/85.5.365

9. Fayers P, Aaronson NK, Bjordal K, Sullivan M. EORTC QLQC30 scoring manual: European Organisation for Research and Treatment of Cancer; 1995.

10.Fernando A, Jayarajah U, Prabashani S, Fernando EA, Seneviratne SA. Incidence trends and patterns of breast cancer in Sri Lanka: an analysis of the national cancer database. BMC cancer. 2018;18[1]:482. https://doi.org/10.1186/s12885-0184408-4

11.Jayarajah U, Udayanga V, Fernando A, Samarasekera DN, Seneviratne $S$. The incidence and patterns of colorectal cancers in Sri Lanka from 2001 to 2010: Analysis of national cancer registry data. European Journal of Cancer Care. 2020:e13247. https://doi.org/10.1111/ecc.13247

12.Jayarajah U, Fernando A, Prabashani S, Fernando EA, Seneviratne SA. Incidence and histological patterns of thyroid cancer in Sri Lanka 2001-2010: an analysis of national cancer registry data. BMC cancer. 2018;18[1]:163.

https://doi.org/10.1186/s12885-018-4083-5

13. Gradishar WJ, Anderson BO, Balassanian R, Blair SL, Burstein HJ, Cyr A, et al. Invasive breast cancer version 1.2016, NCCN clinical practice guidelines in oncology. Journal of the National Comprehensive Cancer Network. 2016;14[3]:324-54. https://doi.org/10.6004/jnccn.2016.0037

14.Excellence. NIfHaC. Early and locally advanced breast cancer: diagnosis and managemene, Evidence reviews for breast radiotherapy. 2018.

15.Feuer EJ, Wun L-M. How much of the recent rise in breast cancer incidence can be explained by increases in mammography utilization? A dynamic population model approach. American Journal of Epidemiology. 1992;136[12]:1423-36. https://doi.org/10.1093/oxfordjournals.aje.a116463

16. Nilaweera R, Perera S, Paranagama N, Anushyanthan A. Knowledge and practices on breast and cervical cancer screening methods among female health care workers: a Sri Lankan experience. Asian Pacific Journal of Cancer Prevention. 2012;13[4]:1193-6.

https://doi.org/10.7314/APJCP.2012.13.4.1193

17.Porter P. "Westernizing" women's risks? Breast cancer in lowerincome countries. New England Journal of Medicine. 2008;358[3]:213-6. https://doi.org/10.1056/NEJMp0708307 
18.Abeykoon A. Ageing and the health sector in Sri Lanka. Ceylon Medical Journal. 2015;45[2].

https://doi.org/10.4038/cmj.v45i2.7999

19. Ferlay J, Soerjomataram I, Dikshit R, Eser S, Mathers C, Rebelo $\mathrm{M}$, et al. Cancer incidence and mortality worldwide: sources, methods and major patterns in GLOBOCAN 2012. International journal of cancer. 2015;136[5]:E359-E86.

https://doi.org/10.1002/ijc.29210
20.Balawardena J, Skandarajah T, Rathnayake W, Joseph N. Breast Cancer Survival in Sri Lanka. JCO Glob Oncol. 2020;6:589-99. https://doi.org/10.1200/JGO.20.00003 